\title{
Effects of Gkn1 On Biofilm Formation
}

Tova Wasserman-Bruck ${ }^{1}$, Toni Boger-May ${ }^{1}$, Derek Rubadeux², Katelyn Ruley Haase ${ }^{1}$, David L. Boone ${ }^{1,2}$

${ }^{1}$ Indiana University School of Medicine, South Bend IN; ${ }^{2}$ Department of Biological Sciences, University of Notre Dame, South Bend, IN

\section{Background and Objectives:}

Gastrokine-1 (Gkn1) is a protein produced solely in the stomach and secreted into the lumen of the gut. Gkn1 has a BRICHOS domain which is anti-amyloidogenic. While the exact function of Gkn1 is not yet completely understood, Gkn1 has a protective role against IBD in induced models of colitis. Since microbes secrete amyloid to facilitate biofilm formation, we hypothesized that Gkn1 may play a protective role against IBD by inhibiting amyloid formation by biofilm forming microbes in the gut. We examined the effects of varying concentrations of Gkn1 on known biofilm forming microbes to determine if there was a dose responsive inhibitory effect of Gkn1 on biofilm formation.

\section{Methods:}

Bacterial colonies from Adherent Invasive E. coli and E. faecalis were cultured and incubated at 37 degrees Celsius overnight. The samples were then diluted 1:100 and inoculated into 96-well costar microassay plates with concentrations of Gkn1 ranging from 0.00075 to $0.025 \mathrm{mg} / \mathrm{ml}$. At 8,24 , or 48 hour time points the plates were then washed to remove planktonic bacteria, and adherent biofilms were stained with crystal violet. Biofilm formation was analyzed by comparing the OD590/OD600 against a control.

\section{Results:}

Using a one-way ANOVA, we determined that there is dose response inhibition of biofilm formation with Gkn1, with the greatest inhibition of biofilm formation at concentrations of Gkn1 between 0.00312 and $0.0125 \mathrm{mg} / \mathrm{ml}$.

\section{Conclusions:}

A dose response inhibition of Gkn1 on biofilm formation was identified. Since Gkn1 has demonstrated a protective role against IBD in induced colitis, targeting intestinal luminal amyloids may be a potential therapeutic approach for IBD. 